\section{ONCO-IMMUNOLOGICAL MECHANISMS OF FOCAL ABLATION AND LOCALIZED IL-12 IMMUNOTHERAPY}

Maura Vrabel ${ }^{*}$, David Zaharoff, Siena Mantooth. NC State University, Raleigh, NC, USA

Background Unresectable solid malignancies are responsible for a major proportion of total cancer-related mortalities, making focal ablation an attractive alternative. Nevertheless, there are high rates of recurrence after ablation [1,2]. The addition of an immune agonist to ablation has the potential to prevent this recurrence and improve treatment outcomes. The goal of this study is to determine if localized interleukin-12 (IL-12) can prevent primary tumor recurrence after cryoablation in both minimal ablation and metastasis models.

Methods LLC (LL/2) (ATCC) and MC38 (NCI) were implanted in 6-9 week old $\mathrm{C} 57 \mathrm{BL} / 6$ mice. All tumors were treated at volumes of 200-500 $\mathrm{mm} 3$. LLC tumors with treated with three cycles of freeze/thaw and then monitored for tumor recurrence and lung metastasis 22-25 days after implantation. MC38 tumors were treated with a minimal cryoablation protocol where the tumor undergoes one cycle of freezing at $100 \%$ intensity up to the tumor margin by visual inspection, followed by one cycle of active thaw until the cryo probe can be removed. Ablation was performed using the Argon-Helium Visual-ICETM Cryoablation System (Boston Scientific). Interleukin-12 (IL-12) in 1.5\% (w/v) chitosan acetate (CS) dissolved in dPBS was injected intratumorally within an hour after cryoablation, or as indicated. The dose of IL-12 was 1 ug unless otherwise indicated.

Results We established a model of $100 \%$ recurrence using the minimal cryoablation protocol. Using this protocol, we demonstrated that a single intratumoral injection of CS/IL-12 within an hour after cryoablation prevents recurrence in $7 / 8$ mice while only $3 / 8$ mice remained tumor-free without CS/IL-12 (Figure 1). Studies evaluating the impact of CS/IL-12 on tumor-specific $\mathrm{T}$ cell responses following cryoablation are ongoing. In the spontaneously metastatic LLC model, delivering CS/IL-12 two days before treatment, either resection or cryoablation, reduced the number of metastatic lung nodules and furthermore prevented the recurrence of the primary tumor after resection (Figure 2).
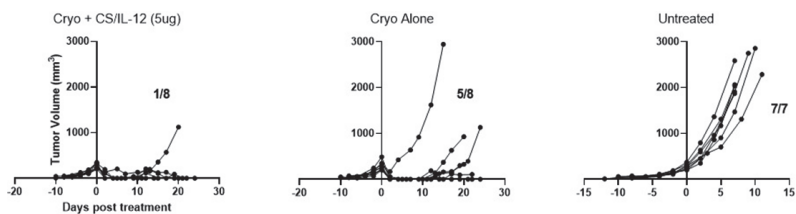

Abstract 620 Figure 1 CS/IL-12 prevents tumor recurrence after minimal cryo
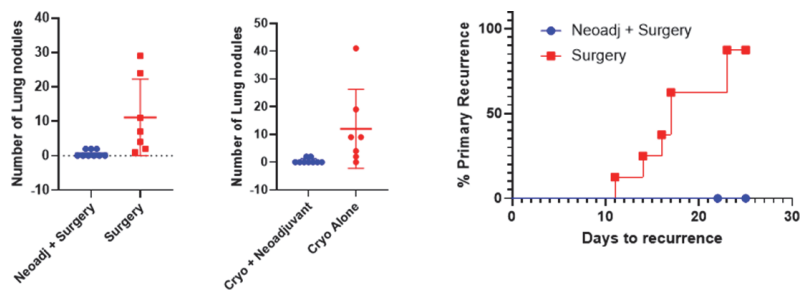

Abstract 620 Figure 2 CS/IL-12 before cryo or surgery prevents metastasis
Conclusions Due to the high percentage of solid malignancies that are unresectable at diagnosis, focal ablation is an attractive alternative, yet has a high rate of recurrence. We demonstrated that intratumoral neoadjuvant CS/IL-12 protects not only against primary recurrence after cryoablation, but also protects against lung metastasis and recurrence after resection. Further studies are necessary to explore the immune populations responsible for this therapeutic effect.

Acknowledgements This work is supported by Boston Scientific, the NC State University Provost's Fellowship, the NSF Graduate Research Fellowship and startup funds provided by the College of Engineering at NC State University.

\section{REFERENCES}

1. Weld KJ, Landman J. Comparison of cryoablation, radiofrequency ablation and high-intensity focused ultrasound for treating small renal tumours. BJU Int 2005:96(9):1224-1229. doi:10.1111/j.1464-410X.2005.05848.x.

2. Guenther $E$, Klein N, Zapf $S$, et al. Prostate cancer treatment with Irreversible Electroporation (IRE): Safety, efficacy and clinical experience in 471 treatments. PloS One. 2019;14(4):e0215093. doi:10.1371/journal.pone.0215093

Ethics Approval The Institutional Animal Care and Use Committee at North Carolina State University approved of all animal protocols (\#19-795) in compliance with The Guide for Care and Use of Laboratory Animals (National Research Council).

http://dx.doi.org/10.1136/jitc-2021-SITC2021.620 